\title{
INVARIANTS OF COADJOINT REPRESENTATIONS OF REGULAR FACTORS
}

\author{
A. N. PANOV
}

\begin{abstract}
Generators are found for the field of invariants of coadjoint representations for the Lie algebras that are factors of a unitriangular Lie algebra by some regular ideal.
\end{abstract}

\section{$\S 0$. INTRODUCTION}

Coadjoint orbits play an important role in representation theory, symplectic geometry, and mathematical physics. In accordance with the orbit method of Kirillov [1, 2, for nilpotent Lie groups there exists a one-to-one correspondence between coadjoint orbits and irreducible representations in Hilbert spaces. This makes it possible to solve problems of representation theory and harmonic analysis in geometrical terms of the orbit space. However, the problem of classification of all coadjoint orbits for specific Lie groups (such as the group of unitriangular matrices) is still open and far from its solution. In the pioneering paper 2 on the orbit method, a description of the algebra of invariants and a classification of orbits of maximal dimension were obtained.

The main result of this paper consists in constructing generators of the field of invariants for the coadjoint representations of regular factors. In what follows, by a regular factor we mean a Lie algebra that is a factor of a unitriangular Lie algebra modulo some regular ideal. The paper consists of three sections. In $\S 1$ we study the diagram method introduced in [3, 4. In $\S 2$ we present the notion of an extremal minor of the characteristic matrix (Definition 2.4). We show that its highest coefficient is invariant with respect to the coadjoint representation (Theorem 2.5). The method of proof is based on reduction of quantum minors. We state Conjecture 2.6 concerning the structure of the algebra of invariants $K\left[\mathcal{L}^{*}\right]^{L}$. In the last $\S 3$ we prove that the field of invariants $K\left(\mathcal{L}^{*}\right)^{L}$ is a field of rational functions on a system of invariants (Theorem 3.20).

Let $N=\mathrm{UT}(n, K)$ be the group of unitriangular matrices of size $n \times n$ with units on the diagonal and with entries in the field $K$ of zero characteristic. The Lie algebra $\mathfrak{n}=\mathfrak{u t}(n, K)$ of this group consists of lower triangular matrices of size $n \times n$ with zeros on the diagonal. One can define the natural representation of the group $N$ in the conjugate space $\mathfrak{n}^{*}$ by the formula $\operatorname{Ad}_{g}^{*} f(x)=f\left(\operatorname{Ad}_{g}^{-1} x\right)$, where $f \in \mathfrak{n}^{*}, x \in \mathfrak{n}$, and $g \in N$. This representation is called the coadjoint representation. We identify the symmetric algebra $S(\mathfrak{n})$ with the algebra of regular functions $K\left[\mathfrak{n}^{*}\right]$ on the conjugate space $\mathfrak{n}^{*}$. Also, we identify $\mathfrak{n}^{*}$ with the subspace of upper triangular matrices with zeros on the diagonal. The pairing of $\mathfrak{n}$ and $\mathfrak{n}^{*}$ is realized by the Killing form $(a, b)=\operatorname{Tr}(a b)$, where $a \in \mathfrak{n}$, $b \in \mathfrak{n}^{*}$. After this identification, the coadjoint action can be realized by the formula

2010 Mathematics Subject Classification. Primary 17B10.

Key words and phrases. Lie algebra, coadjoint representation, algebra of invariants.

Supported by RFBR (grant nos. 08-01-00151-a, 09-01-00058-a), and by ADTP (grant no. 3341). 
$\operatorname{Ad}_{g}^{*} b=P\left(\operatorname{Ad}_{g} b\right)$, where $P$ is the natural projection of the space of $(n \times n)$-matrices onto $\mathfrak{n}^{*}$.

Recall that for any Lie algebra $\mathfrak{g}$ the algebra $K\left[\mathfrak{g}^{*}\right]$ is a Poisson algebra with respect to the Poissson bracket such that $\{x, y\}=[x, y]$ for any $x, y \in \mathfrak{g}$. In the case where $k=\mathbb{R}$, the symplectic leaves with respect to this Poisson bracket coincide with the orbits of the coadjoint representation [1. Accordingly, the algebra of Casimir elements in $K\left[\mathfrak{g}^{*}\right]$ coincides with the algebra of invariants $K\left[\mathfrak{g}^{*}\right]^{N}$ of the coadjoint representation.

The coadjoint orbits of the group $N$ are closed in Zariski topology in $\mathfrak{n}^{*}$ because all orbits of a regular action of an arbitrary algebraic unipotent group in an affine algebraic variety are closed; see [7, 11.2.4].

To simplify our language, we give the following definition: a root is an arbitrary pair $(i, j)$, where $i, j$ are positive integers from 1 to $n$ and $i \neq j$. The permutation group $S_{n}$ acts on the set of roots by the formula $w(i, j)=(w(i), w(j))$.

A root $(i, j)$ is positive if $i>j$. Respectively, a root is negative if $i<j$. We denote the set positive roots by $\Delta_{+}$.

For any root $\eta=(i, j)$ we denote by $-\eta$ the $\operatorname{root}(j, i)$. We define the partial operation of addition on the set of positive roots: if $\eta=(i, j) \in \Delta_{+}$and $\eta^{\prime}=(j, m) \in \Delta_{+}$, then $\eta+\eta^{\prime}=(i, m)$.

Consider the standard basis $\left\{y_{i j}:(i, j) \in \Delta_{+}\right\}$in the algebra $\mathfrak{n}$. We also use the notation $y_{\xi}$ for $y_{i j}$, where $\xi=(i, j)$.

An ideal $\mathfrak{m}$ in the Lie algebra $\mathfrak{n}$ is said to be regular if it is generated by a subsystem of vectors of the standard basis. Then $\mathfrak{m}=\operatorname{span}\left\{y_{\eta} \mid \eta \in M\right\}$, where $M$ is a subset of $\Delta_{+}$with the following property: in a sum of two positive roots, if one of the summands belongs to $M$, then the sum also belongs to $M$.

Denote by $\mathcal{L}$ the Lie factor algebra $\mathfrak{n} / \mathfrak{m}$ (the regular factor) and by $L$ the corresponding factor group of $N$ with respect to the normal subgroup $\exp (\mathfrak{m})$. Note that the conjugate space $\mathcal{L}^{*}$ is the subspace in $\mathfrak{n}^{*}$ that consists of all $f \in \mathfrak{n}^{*}$ annihilating $\mathfrak{m}$. The coadjoint $L$-orbit for $f \in \mathcal{L}^{*}$ coincides with its $N$-orbit.

\section{§1. Diagram and permutation associated with a Lie algebra $\mathcal{L}$}

In the paper [3], to any regular factor $\mathcal{L}$ we assigned a diagram $\mathcal{D}_{\mathcal{L}}$ constructed by applying some formal rule of arrangement of symbols in the table. By using the diagram $\mathcal{D}_{\mathcal{L}}$ one can easily calculate the index of the Lie algebra $\mathcal{L}$. Recall that the index of a Lie algebra is the minimum dimension of the centralizer of a linear form on this Lie algebra. For algebraic Lie algebras, the index is equal to the transcendental degree of the field of invariants of the coadjoint representation. For nilpotent Lie algebras (for example, $\mathfrak{u t}(n, K))$, the field of invariants of the coadjoint representation is the pure transcendental extension of the ground field of degree equal to the index [7. Accordingly, using the diagram one can easily calculate the maximum dimension of coadjoint orbits (see Theorem 1.2). Earlier, the diagram method was used for a classification of all coadjoint orbits of the unitriangular group of size $n \leq 7$ [5], and for a description of special families of coadjoint orbits for an arbitrary $n$ (the subregular orbits [5]; the orbits associated with involutions [6]).

Now we describe the construction of $\mathcal{D}_{\mathcal{L}}$ and formulate the main assertions of the papers [3, 4]. Consider the order $\succ$ on the set $\Delta_{+}$such that

$$
(n, 1) \succ(n-1,1) \succ \cdots \succ(2,1) \succ(n, 2) \succ \cdots \succ(3,2) \succ \cdots \succ(n, n-1) .
$$

Given an ideal $\mathfrak{m}$, we construct a diagram that is an $(n \times n)$-matrix in which all places $(i, j)$ with $i \leq j$ are not filled and all other places (i.e., the places of $\Delta_{+}$) are filled with the symbols " $\otimes$ ", "•", "+", and "-" according to the following rules. The places 
$(i, j) \in M$ are filled with the symbol "•". We refer to the procedure of putting "•" to the places in $M$ as the zeroth step in the construction of the diagram.

We put the symbol " $\otimes$ " in the greatest (in the sense of the order $\succ$ ) place in $\Delta_{+} \backslash M$. Note that this symbol will occupy a place in the first column if the set of pairs of the form $(i, 1)$ in $\Delta_{+} \backslash M$ in nonempty. Suppose that we put the symbol " $\otimes$ " in the place $(k, t), k>t$. Next, we put the symbol "-" in all places $(k, a), t<a<k$, and we put the symbol "+" in all places $(b, t), 1<b<k$. This procedure finishes the first step of constructing the diagram.

After that, we put the symbol " $\otimes$ " in the greatest (in the sense of the order $\succ$ ) empty place in $\Delta_{+}$. As above, we put the symbols "+" and "-" in empty places, taking into account the following: we put the symbols "+" and "-" in pairs; if both places $(k, a)$ and $(a, t)$, where $k>a>t$, are empty, we put "-" in the first place and "+" in the second; if one of these places, $(k, a)$ or $(a, t)$, is already filled, then we do not fill the other place. After this procedure we finish the second step.

Continuing in this way, we obtain a diagram. We denote this diagram by $\mathcal{D}_{\mathcal{L}}$. The number of the last step is equal to the number of the symbols " $\otimes$ " in the diagram.

Example 1. Let $n=7, \mathfrak{m}=K y_{51} \oplus K y_{61} \oplus K y_{71} \oplus K y_{62}$. The corresponding diagram is as follows:

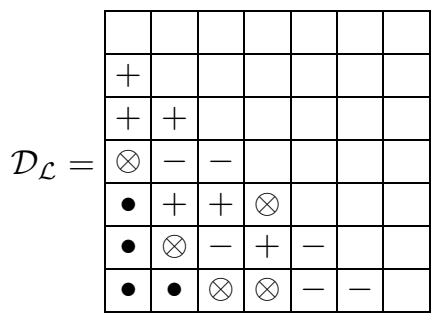

We construct this diagram in five steps, beginning with the zeroth step:
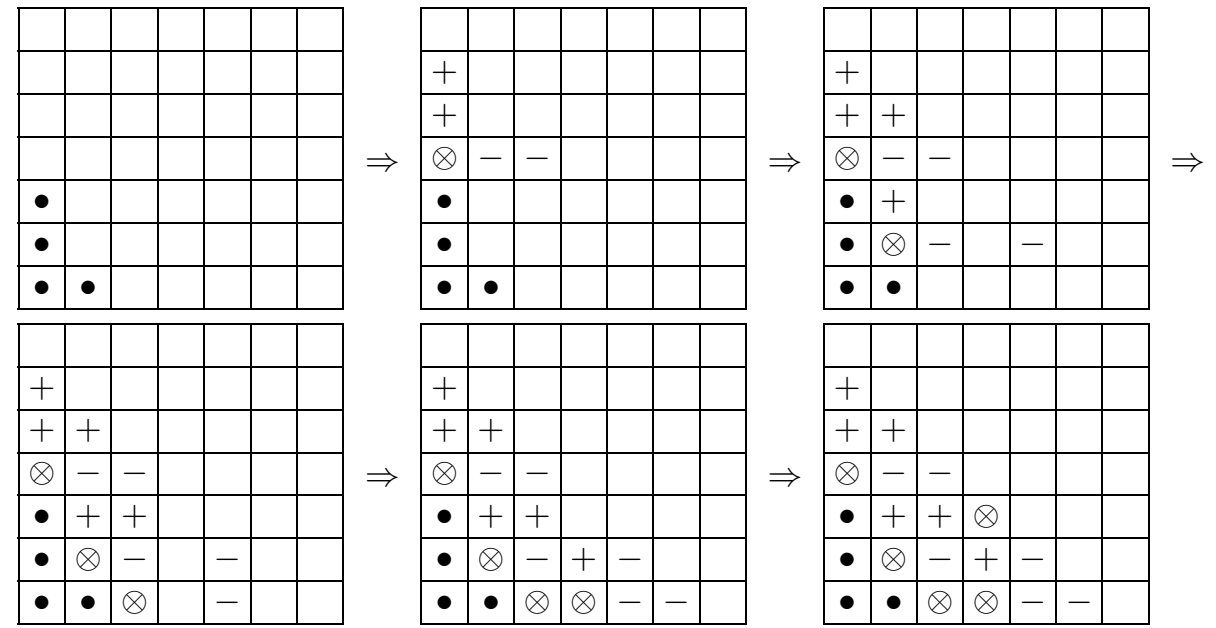

We denote by $S=\left\{\xi_{1} \succ \xi_{2} \succ \cdots \succ \xi_{s}\right\}$ the set of pairs $(i, j)$ filled with the symbol " $\otimes$ " in the diagram. For the diagram of Example 1, we have $S=\left\{\xi_{1} \succ \xi_{2} \succ \xi_{3} \succ \xi_{4} \succ \xi_{5}\right\}$, where $\xi_{1}=(4,1), \xi_{2}=(6,2), \xi_{3}=(7,3), \xi_{4}=(7,4), \xi_{5}=(5,4)$.

Let $\mathbb{A}_{m}$ denote the Poisson algebra $K\left[p_{1}, \ldots, p_{m} ; q_{1}, \ldots, q_{m}\right]$ with the bracket $\left\{p_{i}, q_{j}\right\}=\delta_{i j}$.

Recall that a Poisson algebra $\mathcal{A}$ is the tensor product of two Poisson algebras $\mathcal{B}_{1} \otimes \mathcal{B}_{2}$ if $\mathcal{A}$ is isomorphic to $\mathcal{B}_{1} \otimes \mathcal{B}_{2}$ as a commutative associative algebra and $\left\{\mathcal{B}_{1}, \mathcal{B}_{2}\right\}=0$. 
Theorem 1.1 [3]. There exist $z_{1}, \ldots, z_{s} \in K\left[\mathcal{L}^{*}\right]^{L}$, where $s=|S|$, with the following properties.

1) For every $i, z_{i}=y_{\xi_{i}} Q_{i}+P_{>i}$, where $Q_{i}$ is a product of powers of $z_{1}, \ldots, z_{i-1}$ and $P_{>i}$ is a polynomial in the variables $\left\{y_{\eta}, \eta \succ \xi_{i}\right\}$.

2) Denote by $\mathcal{Z}$ the set of denominators generated by $z_{1}, \ldots, z_{s}$; the localization $K\left[\mathcal{L}^{*}\right]_{\mathcal{Z}}$ of the algebra $K\left[\mathcal{L}^{*}\right]$ with respect to the set of denominators $\mathcal{Z}$ is isomorphic as a Poisson algebra to the tensor product $K\left[z_{1}^{ \pm}, \ldots, z_{s}^{ \pm}\right] \otimes \mathbb{A}_{m}$ for some $m$.

Theorem 1.1 directly implies the following statement.

Theorem 1.2 [3. 1) The field of invariants $K\left(\mathcal{L}^{*}\right)^{L}$ coincides with $K\left(z_{1}, \ldots, z_{s}\right)$.

2) The maximum dimension of a coadjoint orbit in $\mathcal{L}^{*}$ is equal to the number of the symbols "+" and "_" in the diagram $\mathcal{D}_{\mathcal{L}}$.

3) The index of the Lie algebra $\mathcal{L}$ coincides with the number of symbols " $\otimes$ " in the diagram $\mathcal{D}_{\mathcal{L}}$

To the Lie algebra $\mathcal{L}$, we assign a permutation defined as follows.

Definition 1.3 . Denote by $w=w_{\mathcal{L}}$ the permutation in $S_{n}$ such that

1) $w(1)=\max \{1 \leq i \leq n \mid(i, 1) \notin M\}$;

2) $w(t)=\max \{1 \leq i \leq n \mid(i, t) \notin M, i \notin\{w(1), \ldots, w(t-1)\}\}$ for all $2 \leq t \leq n$.

As usual, we denote by $l(w)$ the minimum number of factors in decompositions of $w$ into products of simple reflections. The number $l(w)$ coincides with the number of inversions in the rearrangement $(w(1), \ldots, w(n))$.

Theorem 1.4 [4, Theorem 2.2]. The number $l(w)$ coincides with $\operatorname{dim} \mathcal{L}$.

Theorem 1.5 [4, Theorem 2.6]. We have $w=r_{\xi_{1}} r_{\xi_{2}} \cdots r_{\xi_{s}}$.

Denote by $\Delta_{+}^{(t)}$ the set of $\eta \in \Delta_{+}$that have the form $(b, t)$ for $b>t$. Let $S^{(t)}=\Delta_{+}^{(t)} \cap S$, and let

$$
S^{[t]}=S^{(1)} \sqcup \cdots \sqcup S^{(t)} .
$$

We denote by $w^{(t)}$ (respectively, $w^{[t]}$ ) the product of reflections $r_{\xi}$ (arranged in the decreasing order, in the sense of $\succ$ ), where $\xi \in S^{(t)}$ (respectively, $S^{[t]}$ ). It is easily seen that

$$
w^{[t]}=w^{(1)} \cdots w^{(t)} .
$$

Theorem 1.6 4, Theorem 2.7]. If $\eta \in A^{(t)}$, then: 0

1) the place $\eta$ in the diagram $\mathcal{D}_{\mathcal{L}}$ is filled with the symbol "-" if and only if $w^{[t-1]}(\eta)<$

2) the place $\eta$ in the diagram $\mathcal{D}_{\mathcal{L}}$ is filled with the symbol "•" if and only if $w^{[t]}(\eta)>0$;

3 ) the place $\eta$ in the diagram $\mathcal{D}_{\mathcal{L}}$ is filled with the symbol "+" or " $\otimes$ " if and only if $w^{[t-1]}(\eta)>0$ and $w^{[t]}(\eta)<0$.

For each $\xi \in S^{(t)}$, we denote by $w_{\xi}$ the product of the reflections $r_{\xi^{\prime}}$ (arranged in decreasing order, in the sense of $\succ$ ), where $\xi^{\prime} \in S$ and $\xi^{\prime} \succeq \xi$. It is easily seen that if $\xi \in \Delta_{+}^{(t)}$, then

$$
w_{\xi}=w^{(1)} \cdots w^{(t-1)} w_{\xi}^{(t)},
$$

where $w_{\xi}^{(t)}$ is the product of the reflections $r_{\xi^{\prime}}$ (arranged in decreasing order, in the sense of $\succ)$, where $\xi^{\prime} \in S^{(t)}$ and $\xi^{\prime} \succeq \xi$. 
Theorem 1.7. Let $\xi=\xi_{m} \in S^{(t)}$ and $\eta \in \Delta_{+}$. Suppose that either a) $\xi \succ \eta$ or b) $\eta \in \Delta_{+}^{(t)}$. We claim that

1) if the place $\eta$ was empty after the mth step or was filled with the symbol "•", then $w_{\xi}(\eta)>0$

2) if the place $\eta$ was filled after the mth step with any symbol other than "•", then $w_{\xi}(\eta)<0$.

Proof. If the place $\eta$ is filled with the symbol $\bullet$, then $w_{\xi}(\eta)>0$ (in case b), see 4 , Proposition 2.5]; in case a), this can be proved similarly). If the place $\eta$ is empty after the $m$ th step, then $w_{\xi}(\eta)>0$ (in both cases a) and b), see [4, Proposition 2.3(1)]). If the place $\eta$ was filled after the $m$ th step with any symbol other than "•", then $w_{\xi}(\eta)<0$ (in case a), see [4. Proposition 2.3(2)]; in case b), one can argue as in the proof of [4, Proposition 2.5(2)]).

Corollary 1.8. For every $\xi \in S$ we have $w_{\xi}(\xi)<0$.

Proof. Let $\xi=\xi_{m}$. Then

$$
w_{\xi}(\xi)=w_{\xi_{m-1}} r_{\xi}(\xi)=-w_{\xi_{m-1}}(\xi)<0
$$

because the place $\xi=\xi_{m}$ was empty after the $(m-1)$ th step.

\section{§2. Reduction of QuAntum Minors}

We recall some definitions of the theory of quantum matrices. Let $q$ be a variable. The algebra of regular functions on quantum matrices (briefly, the algebra of quantum matrices) $K_{q}[\operatorname{Mat}(n)]$ is generated by the elements $\left\{a_{i j}\right\}_{i, j=1}^{n}$ subject to the system of relations $a b=q b a, c d=q d c, a c=q c a, b d=q d b, b c=c b, a d-d a=\left(q-q^{-1}\right) b c$, imposed on any $(2 \times 2)$-submatrix $\left(\begin{array}{ll}a & b \\ c & d\end{array}\right)$. The algebra $K_{q}[\operatorname{Mat}(n)]$ is a Noetherian ring without zero divisors and with the Gelfand-Kirillov dimension equal to $n^{2}$ (see, e.g., 8]).

Denote by $B:=B_{-}$the group of lower triangular matrices and by $\mathfrak{b}:=\mathfrak{b}_{-}$its Lie algebra. The algebra of regular functions $K_{q}[B]$ on the quantum group $B$ can be constructed by factorizing the algebra $K_{q}[\operatorname{Mat}(n)]$ modulo the ideal $\left\langle a_{i j} \mid i<j\right\rangle$, followed by localization with respect to the set of denominators generated by $a_{11}, \ldots, a_{n n}$.

The quantum analog of the ideal $\mathfrak{m}$ in the Lie algebra $\mathfrak{n}$ is the ideal $Q_{\mathfrak{m}}$ in $K_{q}[B]$ generated by the $a_{i j},(i, j) \in M$. The factor algebra of $K_{q}[B]$ modulo the ideal $Q_{\mathfrak{m}}$ is denoted by $K_{q}[L]$ and is called the algebra of regular functions on the quantum group $L$.

For any systems of columns $J=\left\{j_{1}<\cdots<j_{m}\right\}$ and rows $I=\left\{i_{1}<\cdots<i_{m}\right\}$, the element

$$
\mathbb{M}_{I}^{J}=\sum_{F=x I}(-q)^{l(x)} a_{f_{1}, j_{1}} \cdots a_{f_{m}, j_{m}},
$$

where $F=\left(f_{1}, \ldots, f_{m}\right)$ and $x \in S_{m}$, is called a quantum minor.

By definition, the quantum universal enveloping algebra $U_{q}(\mathfrak{b})$ is generated by elements $Y_{1}, \ldots, Y_{n-1}$ and $K_{1}, \ldots, K_{n}$ subject to the relations

$$
Y_{i} Y_{j}^{2}-\left(q+q^{-1}\right) Y_{i} Y_{j} Y_{i}+Y_{j}^{2} Y_{i}=0
$$

(the quantum Serre relations), where $|j-i|=1$, and

$$
K_{i} Y_{j}=q^{-\left(\varepsilon_{i}, \varepsilon_{j}-\varepsilon_{j+1}\right)} Y_{j} K_{i},
$$

where $1 \leq i \leq n, 1 \leq j \leq n-1$. By the quantum algebra $U_{q}(\mathfrak{n})$ we mean the subalgebra of $U_{q}(\mathfrak{b})$ generated by $\left\{Y_{i}\right\}$. The ideal $\mathfrak{m}$ corresponds to the ideal $\widetilde{Q}_{\mathfrak{m}}$ in $U_{q}(\mathfrak{n})$ generated by all $Y_{i j}$, where $i>j$ and $(i, j) \in M$. We say that the quantum group $U_{q}(\mathcal{L})$ is the factor algebra of $U_{q}(\mathfrak{n})$ modulo the ideal $\widetilde{Q}_{\mathfrak{m}}$. 
The universal enveloping algebra $U(\mathfrak{n})$ is the factor algebra of $U_{q}(\mathfrak{n})$ modulo $q-1$. The symmetric algebra $S(\mathfrak{n})$ coincides with the graded algebra $\operatorname{gr} U(\mathfrak{n})$. Similarly, for the Lie algebra $\mathcal{L}$ we have

$$
S(\mathcal{L})=\operatorname{gr}\left(U_{q}(\mathcal{L}) \bmod (q-1)\right) .
$$

It is obvious that the algebras $K_{q}[B]$ and $U_{q}(\mathfrak{b})$ are not isomorphic (because their factors modulo $q-1$ are not isomorphic). We denote by $K_{q}^{\prime}[B]$ and $U_{q}^{\prime}(\mathfrak{b})$ the localizations of $K_{q}[B]$ and $U_{q}(\mathfrak{b})$ modulo $q-1$.

It is well known that the algebras $K_{q}^{\prime}[B]$ and $U_{q}^{\prime}(\mathfrak{b})$ are isomorphic. This isomorphism is called the Drinfeld isomorphism. It can be constructed directly: the subalgebra of $K_{q}^{\prime}[B]$ generated by the elements

$$
Y_{i j}=-\frac{a_{i j} a_{j j}^{-1}}{q-q^{-1}}, \quad \text { where } i>j, \quad \text { and } \quad K_{i}=a_{i i}^{-1}, \quad \text { where } 1 \leq i \leq n,
$$

is isomorphic to $U_{q}^{\prime}(\mathfrak{b})$ and coincides with $K_{q}^{\prime}[B]$ (see, e.g., [9]). Briefly, this can be checked as follows: we show that $\left\{Y_{i+1, i}, 1 \leq i \leq n-1\right\}$ satisfy the quantum Serre relations; we extend the correspondence $Y_{i} \mapsto Y_{i+1, i}$ to a homomorphism of $U_{q}(\mathfrak{b})$ into $K_{q}^{\prime}[B]$; this homomorphism is an isomorphism, because it induces an isomorphism of the corresponding graded algebras.

In what follows, we identify $U_{q}(\mathfrak{b})$ and $U_{q}(\mathfrak{n})$ with their images in $K_{q}^{\prime}[B]$. Note that the system of ordered (lexicographically) monomials in $\left\{Y_{i j} \mid i>j\right\}$ is a basis of $U_{q}(\mathfrak{n})$ as a free module over $K\left[q, q^{-1}\right]$.

Consider the right action of the $n$-dimensional torus on the algebra of quantum matrices by the formula $a_{i j} \cdot \mathbf{t}=t_{j} a_{i j}$, where $\mathbf{t}=\left(t_{1}, \ldots, t_{n}\right)$. We say that an element $b$ of $K_{q}[B]$ is homogeneous of weight $\left(k_{1}, \ldots, k_{n}\right)$ if

$$
\text { b. } \mathbf{t}=t_{1}^{k_{1}} \cdots t_{n}^{k_{n}} b .
$$

Given a homogeneous element $b \in K_{q}[B]$, we construct the element

$$
\widetilde{b}=\frac{(-1)^{k}}{\left(q-q^{-1}\right)^{k}} \cdot b a_{11}^{-k_{1}} \cdots a_{n n}^{-k_{n}}
$$

in $U_{q}^{\prime}(\mathfrak{n})$, where $k=k_{1}+\cdots+k_{n}$.

For the quantum minor $\mathbb{M}_{I}^{J}$ of size $m$ with systems $I$ and $J$ of columns and rows, we obtain

$$
\tilde{\mathbb{M}}_{I}^{J}=\sum_{\substack{F=x I \\ f_{\alpha}>j_{\alpha} \forall 1 \leq \alpha \leq m}}(-q)^{l(x)} q^{\phi(x)}\left(q^{-1}-q\right)^{-\delta(F, J)} Y_{f_{1}, j_{1}} \cdots Y_{f_{m}, j_{m}},
$$

where $\phi(x)$ is an integer and $\delta(F, J)=\operatorname{card}\left\{1 \leq \alpha \leq m \mid f_{\alpha}=j_{\alpha}\right\}$.

We introduce the formal matrix $\Phi_{\mathcal{L}}$ such that all places in $M$ and also the places on and above the diagonal are filled with zeros; on the other places of the form $(i, j), i>j$, we put the elements $y_{i j}$ of the standard basis. For instance, for the Lie algebra $\mathcal{L}$ of Example 1 we obtain the following diagram $\mathcal{D}_{\mathcal{L}}$ and matrix $\Phi_{\mathcal{L}}$ :

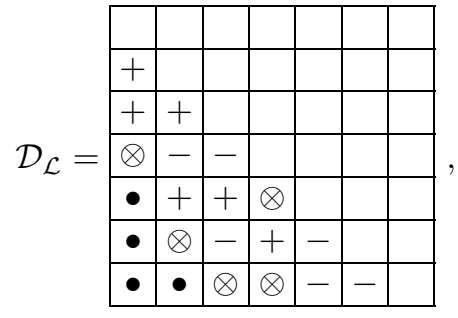

$$
\Phi_{\mathcal{L}}=\left(\begin{array}{ccccccc}
0 & 0 & 0 & 0 & 0 & 0 & 0 \\
y_{21} & 0 & 0 & 0 & 0 & 0 & 0 \\
y_{31} & y_{32} & 0 & 0 & 0 & 0 & 0 \\
y_{41} & y_{42} & y_{43} & 0 & 0 & 0 & 0 \\
0 & y_{52} & y_{53} & y_{54} & 0 & 0 & 0 \\
0 & y_{62} & y_{63} & y_{64} & y_{65} & 0 & 0 \\
0 & 0 & y_{73} & y_{74} & y_{75} & y_{76} & 0
\end{array}\right) .
$$


Let $\lambda$ be a variable. Any minor $M_{I}^{J}$ of the matrix $\Phi_{\mathcal{L}}$ is an element of $S(\mathcal{L})=K\left[\mathcal{L}^{*}\right]$. Consider the characteristic matrix $\Phi_{\mathcal{L}}-\lambda E$. The minor $M_{I}^{J}(\lambda)$ of the characteristic matrix with systems $I$ and $J$ of rows and columns has the form

$$
M_{I}^{J}(\lambda)=\sum_{\substack{F=x I \\ f_{\alpha}>j_{\alpha} \forall 1 \leq \alpha \leq m}}(-1)^{l(x)}(-\lambda)^{\delta(F, J)} y_{f_{1}, j_{1}} \cdots y_{f_{m}, j_{m}} .
$$

Expanding (2.2) (respectively (2.3)) in powers of $\left(q-q^{-1}\right)^{-1}$ (respectively $\lambda$ ), we obtain

$$
\begin{aligned}
\tilde{\mathbb{M}}_{I}^{J} & =\sum_{\alpha=0}^{n} \mathbb{M}_{\alpha}\left(q-q^{-1}\right)^{-\alpha}, \\
M_{I}^{J}(\lambda) & =\sum_{\alpha=0}^{n} M_{\alpha} \lambda^{\alpha},
\end{aligned}
$$

where $\mathbb{M}_{\alpha} \in U_{q}(\mathfrak{n}), M_{\alpha} \in U(\mathfrak{n})$ for any $\alpha$.

The degree of the quantum minor $\mathbb{M}_{I}^{J}$ (more precisely, the degree modulo $\mathfrak{m}$ ) is defined as the greatest number $d$ such that $\mathbb{M}_{\alpha} \notin \widetilde{Q}_{\mathfrak{m}}$. The degree of $M_{I}^{J}(\lambda)$ is defined in the usual way.

Lemma 2.1. Let $\bar{Q}_{\mathfrak{m}}$ be the ideal of $U_{q}(\mathfrak{n})$ generated by $\widetilde{Q}_{\mathfrak{m}}$ and $q-1$. Then

1) $M_{\alpha}=\operatorname{gr}\left(\mathbb{M}_{\alpha} \bmod \bar{Q}_{\mathfrak{m}}\right)$;

2) the degrees of the minors $\mathbb{M}_{I}^{J}$ and $M_{I}^{J}(\lambda)$ coincide.

Proof. Proof of 1 ) is obvious. We prove 2). By the PBW theorem, the monomials

$$
Y_{f_{1}, j_{1}}^{k_{1}} \cdots Y_{f_{N}, j_{N}}^{k_{N}}
$$

where $k_{1}, \ldots, k_{N} \in \mathbb{Z}_{+}$and $\left(f_{1}, j_{1}\right) \succ \cdots \succ\left(f_{N}, j_{N}\right)$, form a basis of $U_{q}(\mathfrak{n})$ over the field $K$. A similar system of polynomials in $\left\{y_{i j}\right\}$ yields a basis of $S(\mathfrak{n})$ over $K$.

The quantum minor (2.2) is presented as a linear combination of basic monomials of the form (2.6). Hence, $\mathbb{M}_{\alpha}$ belongs to $\widetilde{Q}_{\mathfrak{m}}$ if and only if every monomial included as a summand in $\mathbb{M}_{\alpha}$ belongs to $\widetilde{Q}_{\mathfrak{m}}$. In its turn, the monomial (2.6) belongs to $\widetilde{Q}_{\mathfrak{m}}$ if and only if it contains at least one element $Y_{f, j}$ of $\widetilde{Q}_{\mathfrak{m}}$. Similar arguments are valid for any coefficient $M_{\alpha}$ occurring in (2.5). Hence, $\mathbb{M}_{\alpha}$ belongs to $\widetilde{Q}_{\mathfrak{m}}$ if and only if $M_{\alpha}$ belongs to $S(\mathfrak{n}) \mathfrak{m}$.

Let $d$ be the common degree of the minors $\mathbb{M}_{I}^{J}$ and $M_{I}^{J}(\lambda)$. Then

$$
M_{I}^{J}(\lambda)=M_{d} \lambda^{d}+M_{d-1} \lambda^{d-1}+\cdots+M_{0},
$$

where $M_{d} \neq 0$, and the element $\widetilde{\mathbb{M}}_{I}^{J}$, which is taken modulo $\widetilde{Q}_{\mathfrak{m}}$, decomposes:

$$
\tilde{\mathbb{M}}_{I}^{J}=\left(q-q^{-1}\right)^{-d}\left(\mathbb{M}_{d}+\left(q-q^{-1}\right) \mathbb{M}_{d-1}+\cdots+\left(q-q^{-1}\right)^{d} \mathbb{M}_{0}\right),
$$

where $\mathbb{M}_{d} \neq 0 \bmod \widetilde{Q}_{\mathfrak{m}}$.

For a quantum minor $\mathbb{M}=\mathbb{M}_{I}^{J}$ and a number $1 \leq i<n$, we denote

$$
\begin{aligned}
\mathbb{M}^{\downarrow} & = \begin{cases}\mathbb{M}_{(I \backslash i) \cup i+1}^{J} & \text { if } i \in I \text { and } i+1 \notin I, \\
0 & \text { otherwise, }\end{cases} \\
\mathbb{M}^{\leftarrow} & = \begin{cases}\mathbb{M}_{I}^{(J \backslash i+1) \cup i} & \text { if } i+1 \in J \text { and } i \notin J, \\
0 & \text { otherwise. }\end{cases}
\end{aligned}
$$

Similarly, we introduce the minors $M^{\leftarrow}(\lambda)$ and $M^{\downarrow}(\lambda)$ for $M_{I}^{J}(\lambda)$. The commutation relations of the algebra of quantum matrices imply the following statement. 
Lemma 2.2. If $\mathbb{M}=\mathbb{M}_{I}^{J}$ and $a=a_{i+1, i}$, then the following identity is true in the ring $K_{q}[B]$ :

$$
\mathbb{M} a-q^{s} a \mathbb{M}=-\left(q-q^{-1}\right) \mathbb{M}^{\leftarrow} a_{i+1, i+1}+\left(q-q^{-1}\right) \mathbb{M}^{\downarrow} a_{i i}
$$

for some $s \in\{0,1,-1\}$.

Proof. This follows directly from [8, Lemmas 4.1.5, 5.1.2]. Also, formula (2.9) can be proved by using the formula for the $R$-matrix (see [10, Chapter 7]).

Definition 2.3. A nonzero minor $M_{I}^{J}(\lambda)$ of the characteristic matrix $\Phi_{\mathcal{L}}-\lambda E$ is extremal if for each $i$ we have $\operatorname{deg} M^{\leftarrow}(\lambda)<\operatorname{deg} M(\lambda)$ and $\operatorname{deg} M^{\downarrow}(\lambda)<\operatorname{deg} M(\lambda)$ (these inequalities are also assumed to be true if $M^{\leftarrow}(\lambda)=0$ or $\left.M^{\downarrow}(\lambda)=0\right)$. Roughly speaking, a minor is extremal if its degree decreases when its rows are shifted down or its columns are shifted to the left.

Remark 2.4. Similarly, one can define an extremal minor of the algebra of quantum matrices. From Lemma 2.1 it follows that a minor $M_{I}^{J}(\lambda)$ is extremal if and only if the corresponding quantum minor $\mathbb{M}_{I}^{J}$ is extremal.

We denote by $P_{I}^{J}$ the leading coefficient $M_{d}$ in the decomposition (2.7) of the minor $M_{I}^{J}(\lambda)$.

Theorem 2.5. The leading coefficient $P_{I}^{J}$ of any extremal minor is invariant with respect to the adjoint (respectively, coadjoint) representation of the group $N$ in $S(\mathfrak{n})$ (respectively, in $\left.K\left[\mathfrak{g}^{*}\right]\right)$.

Proof. It suffices to prove that $\operatorname{ad}_{y} P_{I}^{J}=0$, where $y=y_{i+1, i}$ and $1 \leq i \leq n-1$. Denote

$$
Y=Y_{i+1, i}=-\frac{a_{i+1, i} a_{i i}^{-1}}{\left(q-q^{-1}\right)} \text {. }
$$

Formula (2.9) implies that

$$
\widetilde{\mathbb{M}} Y-q^{s} Y \widetilde{\mathbb{M}}=\widetilde{\mathbb{M}} \leftarrow-\widetilde{\mathbb{M}} .
$$

Taking (2.10) modulo $\widetilde{Q}_{\mathfrak{m}}$, we obtain

$$
\begin{aligned}
& \left(q-q^{-1}\right)^{-d}\left\{\left[\mathbb{M}_{d}+\left(q-q^{-1}\right) \mathbb{M}_{d-1}+\cdots\right] Y-Y\left[\mathbb{M}_{d}+\left(q-q^{-1}\right) \mathbb{M}_{d-1}+\cdots\right]\right\} \\
& \quad=\left(q-q^{-1}\right)^{-d}\left\{\left[\mathbb{M}_{d}^{\leftarrow}+\left(q-q^{-1}\right) \mathbb{M}_{d-1}^{\leftarrow}+\cdots\right]-\left[\mathbb{M}_{d}^{\downarrow}+\left(q-q^{-1}\right) \mathbb{M}_{d-1}^{\downarrow}+\cdots\right]\right\}
\end{aligned}
$$

We cancel $\left(q-q^{-1}\right)^{-d}$. Since $\mathbb{M}_{I}^{J}$ is an extremal minor (see Remark 2.4), we have $\mathbb{M}_{d}^{\leftarrow}=\mathbb{M}_{d}^{\downarrow}=0 \bmod \widetilde{Q}_{\mathfrak{m}}$. Next, after reduction modulo $q-1$, we see that $\operatorname{ad}_{y}$ annihilates $\mathbb{M}_{d} \bmod (q-1) \in U(\mathfrak{n})$. Since

$$
P_{I}^{J}=\operatorname{gr}\left(\mathbb{M}_{d} \bmod (q-1)\right),
$$

we have $\operatorname{ad}_{y} P_{I}^{J}=0$.

Conjecture 2.6. The algebra of invariants $K\left[\mathcal{L}^{*}\right]^{L}$ of the coadjoint representation of a regular factor $\mathcal{L}$ is generated by the leading coefficients of the extremal minors.

This conjecture is true if $\mathcal{L}=\mathfrak{n}$ (i.e., $\mathfrak{m}=0$ ). By a corner minor $M_{i}$ we mean a minor of the matrix $\Phi$ that lies on the intersection of the first $i$ columns and last $i$ rows. The algebra of invariants $K[\mathfrak{n}]^{N}$ is generated by the system of corner minors $M_{i}$ (see [2]), where $1 \leq i \leq\left[\frac{n}{2}\right]$, each of which is extremal. 


\section{$\S 3$. FIELD OF INVARIANTS}

In this section, to any $\xi \in S$ we assign an extremal minor $M_{\xi}(\lambda)$ (see Definition 3.8). As was shown in $\S 2$, its leading coefficient $P_{\xi}$ is an invariant of the coadjoint representation of $\mathcal{L}$.

We prove that the field of invariants $K\left(\mathcal{L}^{*}\right)^{L}$ coincides with the field of rational functions in $P_{\xi}$, where $\xi \in S$ (see Theorem 3.20).

Let $\xi$ be an element of $S$, say $\xi=(k, t) \in S$, where $k>t$. As before, $w_{\xi}$ is defined as in (1.3).

Lemma 3.1. Suppose $i>t$. Then:

1) if $r_{\eta}(i)=i$ for each $\eta \in S$ and $\eta \succeq \xi$, then $w_{\xi}(i)=i$;

2) if $r_{\eta}(i) \neq i$ for some $\eta \in S$ and $\eta \succeq \xi$, then $w_{\xi}(i) \leq t$; in particular, $w_{\xi}(i)<i$;

3) $w_{\xi}(i) \leq i$.

Proof. Statement 1) is obvious; statement 3) follows from 1) and 2). To prove 2) we consider the sequence $i_{0}=i, i_{1}=w_{\xi}^{(t)}\left(i_{0}\right)$, and $i_{\alpha}=w^{(t-\alpha+1)}\left(i_{\alpha-1}\right)$, where $2 \leq \alpha \leq t$.

a) Under the assumption of statement 2), we show that there exists $1 \leq \alpha \leq t$ such that $i_{\alpha}=t-\alpha+1$.

Let $1 \leq \alpha \leq t$ be the least number such that the root $\eta$ equal to $(i, t-\alpha+1)$ is contained in $S$ and $\eta \succeq \xi$. Then $i_{\alpha-1}=\cdots=i_{0}=i$.

If the set $\left\{\theta \in S^{(t-\alpha+1)} \mid \theta \succ \eta\right\}$ is empty, then $i_{\alpha}=r_{\eta}(i)=t-\alpha+1$. Otherwise, let $\theta=(j, t-\alpha+1)$ be the smallest, in the sense of $\succ$, element of $S^{(t-\alpha+1)}$ such that $\theta \succ \eta$. Then $j>i$ and $j=w^{(t-\alpha+1)} i_{\alpha-1}=r_{\theta} r_{\eta} i_{\alpha-1}=i_{\alpha}$. The positive root $\theta$ is the sum of two positive roots $\eta$ and $\gamma=(j, i)$. Since $r_{\theta}(\theta)<0$ (see Corollary 1.8) and $r_{\theta}(\eta)>0$ (see Theorem 1.71 )), we have $r_{\theta}(\gamma)<0$. Theorem 1.72 ) implies that the place $\gamma$ had been filled with the symbol "_" before the symbol " $\otimes$ " appeared in the place $\theta$. Hence, there exists at least one symbol " $\otimes$ " in the row $j=i_{\alpha}$ and in the columns with numbers less than or equal to $t-\alpha+1$. Let $\alpha^{\prime}$ be the least number such that $\alpha^{\prime}>\alpha$ and the root $\eta^{\prime}$ equal to $\left(i_{\alpha}, t-\alpha^{\prime}+1\right)$ is contained in $S$. Then $i_{\alpha^{\prime}-1}=\cdots=i_{\alpha}$.

If the set $\left\{\theta \in S^{\left(t-\alpha^{\prime}+1\right)} \mid \theta \succ \eta^{\prime}\right\}$ is empty, then

$$
i_{\alpha^{\prime}}=r_{\eta^{\prime}}\left(i_{\alpha}\right)=t-\alpha^{\prime}+1 .
$$

Otherwise, we can argue as before to show the existence of $\alpha^{\prime \prime}>\alpha^{\prime}>\alpha$ such that the root $\eta^{\prime \prime}$ equal to $\left(i_{\alpha^{\prime}}, t-\alpha^{\prime \prime}+1\right)$ belongs to $S$. Continuing this process, we see that at a $p$ th step the set

$$
\left\{\theta \in S^{\left(t-\alpha^{(p)}+1\right)} \mid \theta \succ \eta^{(p)}\right\}
$$

will be empty, and therefore

$$
i_{\alpha^{(p)}}=t-\alpha^{(p)}+1 .
$$

Note that for the resulting $\alpha=\alpha^{(p)}$ we have $i_{\alpha}=t-\alpha+1<t$ and $i_{\alpha-1} \geq i_{\alpha-2} \geq \cdots \geq i$.

b) Now we finish the proof of statement 2) using induction on the number of elements in the set $\{\eta \in S \mid \eta \succeq \xi\}$. The proof is obvious if this set consists of one element. In the general case, for $1 \leq \alpha \leq t$ as in a) we obtain

$$
w_{\xi}(i)=w^{(1)} \cdots w^{(t-\alpha)} w^{(t-\alpha+1)} \cdots w^{(t-1)} w_{\xi}(t)(i)=w^{[t-\alpha]} i_{\alpha}=w^{[t-\alpha]}(t-\alpha+1) .
$$

By the induction assumption, $w^{[t-\alpha]}(t-\alpha+1) \leq t-\alpha+1<t$. We conclude that $w_{\xi}(i)<t$.

Corollary 3.2. As before, let $\xi=(k, t), k>t$. Then:

1) if $r_{\eta}(k)=k$ for some $\eta \in S$ and $\eta \succeq \xi$, then $w_{\xi}(t)=k>t$;

2) if $r_{\eta}(k) \neq k$ for some $\eta \in S$ and $\eta \succeq \xi$, then $w_{\xi}(t)<t$. 
Proof. Since $\xi \in S$, we have $\xi=\xi_{m}$ for some $1 \leq m \leq s$ and

$$
w_{\xi}(t)=w_{\xi_{m}}(t)=w_{\xi_{m-1}} r_{\xi_{m}}(t)=w_{\xi_{m-1}}(k) .
$$

Applying Lemma 3.1 with $\xi=\xi_{m-1}$ and $i=k$, we complete the proof.

Corollary 3.3. Let $\xi$ be as in the preceding corollary. If $\xi$ is the smallest, in the sense of $\succ$, element of $S^{(t)}$, then:

1) if $r_{\eta}(k)=k$ for some $\eta \in S^{[t-1]}$ (i.e., there is no symbol " $\otimes$ " in the $k$ th row and in the columns with numbers less than $t$ of the diagram $\left.D_{\mathcal{L}}\right)$, then $w(t)=k$;

2) if $r_{\eta}(k) \neq k$ for some $\eta \in S^{[t-1]}$, then $w(t)<t<k$.

Proof. For the smallest, in the sense of $\succ$, root $\xi \in S^{[t-1]}$ we have $w_{\xi}=w^{[t]}$. The statement follows from Corollary 3.2 because $w^{[t]}(j)=w(j)$ for any $1 \leq j \leq t$.

Corollary 3.4. Any symbol " $\otimes$ " of an arbitrary th column is located either in the place $(w(t), t)$ or below it.

Proof. Let $\xi$ be the smallest, in the sense of $\succ$, element of $S^{(t)}$. If $r_{\eta}(k)=k$ for each $\eta \in S^{[t-1]}$, then $(w(t), t)=\xi$. If $r_{\eta}(k) \neq k$ for some $\eta \in S^{[t-1]}$, then $w(t)<t$, the place $(w(t), t)$ is above the diagonal, and therefore above all the symbols " $\otimes$ ".

As before, let $\xi=(k, t), k>t$. Denote

$$
h:=w_{\xi}(t) .
$$

We give the definition of the systems $J$ and $I$ of columns and rows of the minor $M_{\xi}$ (see Definition 3.8) in each of the following cases separately: 1) $h>t$ and 2) $h<t$. By Corollary 3.2, the case where $h=t$ is impossible.

Case 1. $h>t$. By Corollary 3.2, $h=k>t$. In this case there is no symbol " $\otimes$ " in the $k$ th row to the left of the place $\xi=(k, t)$. We put

$$
J:=J(\xi)=\left\{1 \leq j \leq t: w_{\xi}(j) \geq h\right\}, \quad I:=I(\xi)=w J(\xi) .
$$

Obviously, $|I|=|J|$.

Case 2. $h<t$. In this case there exists at least one symbol " $\otimes$ " in the $k$ th row to the left of the place $\xi=(k, t)$. The system $J:=J(\xi)$ is defined as in (3.1). We denote

$$
\begin{aligned}
I_{*} & :=I_{*}(\xi)=\left\{t<i \leq n: i>t, w_{\xi}(i)<h\right\}, \\
I & :=I(\xi)=[h, t] \sqcup I_{*} .
\end{aligned}
$$

Here $[h, t]$ is a segment of positive integers (see Definition 3.12(1)). Note that in Case 2 the fact that $|I|=|J|$ is not obvious beforehand and will be proved in Lemma 3.7.

Remark 3.5. 1) In both cases, $w_{\xi}(j)=w(j)$ for any $1 \leq j<t$. Hence,

$$
J=\{1 \leq j<t: w(j)<h\} \sqcup\{t\} .
$$

2) Let $\xi=\xi_{m}$. An element $i$ belongs to $I_{*}$ (see Case 2) if and only if $w_{\xi}(i, t)<0$. By Theorem 1.7, this is equivalent to the statement that the place $(i, t)$ is filled after the $m$ th step with one of the symbols " $\otimes$ ", "+", or "-" (but not "•").

Lemma 3.6. As before, let $\xi=\xi_{m}=(k, t)$. In both Cases 1 and 2 ,

1) there exists $1 \leq c \leq h$ such that $J=[c, t]$;

2 ) the rectangle $[h, n] \times[1, c)$ in filled with the symbols "•" in the diagram $\mathcal{D}_{\mathcal{L}}$ (respectively, by zeros in the matrix $\Phi_{\mathcal{L}}$ );

3) there are no symbols " $\otimes$ " in the rectangle $[1, h) \times J$ of the diagram $\mathcal{D}_{\mathcal{L}}$. 
Proof. Let $w_{\xi}(j)<h$ for some $1 \leq j<t$. Since $h=w_{\xi}(t)$, we have

$$
h \notin\left\{w_{\xi}(1), \ldots, w_{\xi}(j-1)\right\} .
$$

By $(3.3), w_{\xi}(j)=w(j)<h$ and $h \notin\{w(1), \ldots, w(j-1)\}$. The definition of $w$ implies that $(h, j) \in M$ (i.e., the place $(h, j)$ is filled in the diagram with the symbol "•"). Since $\mathfrak{m}$ is an ideal in $\mathfrak{n}$, the rectangle $[h, n] \times[1, j]$ is contained in $M$. This implies 1 ) and 2).

Since $w(j)>h$ for some $j \in[c, t)$, Corollary 3.4 shows that all symbols " $\otimes$ " that lie in the columns $[c, t)$ are contained in the rows $[h, n]$. This proves 3$)$.

Lemma 3.7. Let $\xi$ be as before, and let $h<t$ (i.e., the situation of Case 2 occurs). Then $|J|=|I|$.

Proof. Let $a^{(t)}$ be the greatest number in $[1, n]$ such that $\left(a^{(t)}, t\right)$ does not belong to $M$. Then $M \cap \Delta_{+}=\left(a^{(t)}, n\right]$. It is easily seen that $w_{\xi}(i)=i$ for any $i \in\left(a^{(t)}, n\right]$. Therefore, $w_{\xi}\left[1, a^{(t)}\right]=\left[1, a^{(t)}\right]$.

Consider two partitions of the segment $\left[1, a^{(t)}\right]$ into two disjoint sets:

$$
\begin{aligned}
& {\left[1, a^{(t)}\right]=[1, t) \sqcup\left[t, a^{(t)}\right],} \\
& {\left[1, a^{(t)}\right]=[1, h) \sqcup\left[h, a^{(t)}\right] .}
\end{aligned}
$$

Recall that $w_{\xi}(t)=h$. We show that

$$
\begin{aligned}
{[1, h) } & =w_{\xi}[1, c) \sqcup w_{\xi}\left(I_{*}\right), \\
{\left[h, a^{(t)}\right] } & =w_{\xi}(J) \sqcup w_{\xi}\left(I_{*}^{\prime}\right),
\end{aligned}
$$

where $I_{*}^{\prime}$ consists of all $i$ such that the place $(i, t)$ was not filled after the $m$ th step.

Since the total cardinalities of the sets on the right and on the left in these formulas are equal, to prove (3.4) and (3.5) it suffices to show that the sets on the right are contained in the corresponding sets on the left.

Indeed, if $i \in I_{*}$, then, by Remark 3.5 and Theorem $1.7 \mathrm{~b}$ ), we have $w_{\xi}(i, t)<0$. Hence, $w_{\xi}(i)<w_{\xi}(t)=h$, whence $w_{\xi}\left(I_{*}\right) \subset[1, h)$.

If $i \in I_{*}^{\prime}$, then, by Theorem $\left.1.7 \mathrm{~b}\right), w_{\xi}(i, t)>0$. Hence, $w_{\xi}(i)>w_{\xi}(t)=h$, so that $w_{\xi}\left(I_{*}^{\prime}\right) \subset\left[h, a^{(t)}\right]$.

The inclusion $w_{\xi}(J) \subset\left[h, a^{(t)}\right]$ follows directly from the definition of $J$. Finally, the inclusion $w_{\xi}[1, c) \subset[1, h)$ follows from Lemma 3.6 2). This proves formulas (3.4) and (3.5).

By (3.4), we have $\left|w_{\xi}\left(I_{*}\right)\right|=h-c$. Then $\left|I_{*}\right|=h-c$. Since $I=I_{*} \sqcup[h, t]$ and $J=[c, t]$, we obtain

$$
I=\left|I_{*}\right|+(t-h+1)=(h-c)+(t-h+1)=t-c+1=|J| .
$$

Definition 3.8. In Cases 1 and 2 , we denote by $M_{\xi}(\lambda)$ the minor of the characteristic matrix $\Phi_{\mathcal{L}}-\lambda E$ with the system of rows $I=I(\xi)$ and system of columns $J=J(\xi)$. We denote by $P_{\xi}$ its leading coefficient.

Our next goal is to prove that, in the two cases, $M_{\xi}(\lambda)$ is an extremal minor; this will be proved in Proposition 3.19. Case 1 is simpler; the reader interested in this case may turn directly to Proposition 3.19. For Case 2 we need to prove several additional statements.

Under the assumptions of Case 2, as before, let $\xi=\xi_{m}=(k, t)$, where $k>t$, and $h=$ $w_{\xi}(t)$, where $h<t$. As in the proof of Lemma 3.7, we define $a^{(t)}:=\max \{i \mid(i, t) \notin M\}$.

Notation 3.9. 1) $E:=\left[h, a^{(t)}\right], F_{0}:=I \backslash J=[c, h)$.

2) $w_{\xi}^{[j]}:=w_{\xi}$ if $j \geq t$, and $w_{\xi}^{[j]}:=w^{[j]}$ if $1 \leq j<t$ (for the definitions of $w^{[j]}$ and $w_{\xi}$ see (1.2) and (1.3)). 
3) We represent $E$ as a union of two disjoint sets $E=F \sqcup D$, where

$$
\begin{aligned}
F & :=\left\{a \in E \mid w_{\xi}^{[a-1]}(a)<h\right\}, \\
D & :=\left\{a \in E \mid w_{\xi}^{[a-1]}(a) \geq h\right\} .
\end{aligned}
$$

4) $w_{\xi *}:=w^{(c)} \cdots w^{(t-1)} w_{\xi}^{(t)}, w_{\xi *}^{[j]}:= \begin{cases}w^{(c)} \cdots w^{(j)} & \text { if } c \leq j<t, \\ w_{\xi *} & \text { if } t<j \leq n .\end{cases}$

Note that $w_{\xi}=w^{[c-1]} w_{\xi *}$. Since $w^{[c-1]}[1, h) \subset[1, h)$ and $w^{[c-1]}(i)=i$ for any $h \leq i \leq n$, we can rewrite (3.6) and (3.7) as follows:

$$
\begin{aligned}
& F=\left\{j \in E \mid w_{\xi *}^{[j-1]}(j) \in F_{0}\right\}, \\
& D=\left\{j \in E \mid w_{\xi *}^{[j-1]}(j) \in J\right\} .
\end{aligned}
$$

Remark 3.10. Note that $h \in D$ (because there are no symbols " $\otimes$ " in the $h$ th row, since otherwise $\left.w_{\xi}(t)<h\right), t \in F$ (because $w_{\xi *}^{[t-1]}(t)=w_{\xi}\left(i_{*}\right) \in F_{0}$, where $i_{*}$ is an element of $I_{*}$ such that $\left(i_{*}, t\right)$ is the greatest, in the sense of $\succ$, element of $S^{(t)}$ ), and $I_{*} \subset F$ (by the definition of $\left.I_{*}\right)$.

Lemma 3.11. As before, let $\xi=(k, t)$, where $k>t$. Suppose $a \in D, b \in F$, and $a>b$. If $(b, p) \in S$, where $p<t$, then $(a, p) \in M$.

Proof. Let $\xi_{l}=(b, p) \in S$. Let $q$ be the greatest number such that $q \leq m$ and $\xi_{q} \in \Delta_{+}^{[a-1]}$. Since $b<a$, we have $l \leq q$.

1) We show that $w_{\xi_{i}}(a) \geq h$ for any $1 \leq i \leq q$. We argue by induction on $i$, starting with the greatest number $q$. Since $a \in D$, for $i=q$ we obtain

$$
w_{\xi_{q}}(a)=w_{\xi}^{[a-1]}(a) \geq h .
$$

Suppose that the inequality $w_{\xi_{i}}(a) \geq h$ has already been proved for a number $i$; we prove it for $i-1$. Let $\xi_{i}=\left(a_{1}, b_{1}\right), a_{1}>b_{1}$. If $a_{1} \neq a$, then $r_{\xi_{i}}(a)=a$, and therefore

$$
w_{\xi_{i}}(a)=w_{\xi_{i-1}} r_{\xi_{i}}(a)=w_{\xi_{i-1}}(a) .
$$

Using the induction assumption, we obtain $w_{\xi_{i-1}}(a) \geq h$. If $a_{1}=a$, then

$$
w_{\xi_{i}}\left(b_{1}\right)=w_{\xi_{i-1}} r_{\xi_{i}}\left(b_{1}\right)=w_{\xi_{i-1}}(a) .
$$

On the other hand, $w_{\xi_{i}}\left(\xi_{i}\right)<0$ (see Corollary 1.8). Since $\xi_{i}=\left(a, b_{1}\right)$, we have

$$
h \leq w_{\xi_{i}}(a)<w_{\xi_{i}}\left(b_{1}\right)=w_{\xi_{i-1}}(a),
$$

which proves statement 1).

2) Now we turn directly to the proof of the lemma. We may assume that $p$ is the greatest number such that $(b, p) \in S$, where $p<t$. Since $b \in F$, we have

$$
w_{\xi_{l}}(b)=w_{\xi}^{[b-1]}(b)<h .
$$

We introduce the notation $\eta=(a, p), \gamma=(a, b)$. Then $\eta=\xi_{l}+\gamma$.

2a) We show that $w_{\xi_{l}}(\gamma)>0$. Indeed, by statement 1) of the proof, we have $w_{\xi_{l}}(a) \geq$ $h$. On the other hand, it was shown above that $w_{\xi_{l}}(b)<h$. We obtain $w_{\xi_{l}}(a)>w_{\xi_{l}}(b)$, whence $w_{\xi_{l}}(\gamma)=w_{\xi_{l}}(a, b)>0$.

2b) Since $w_{\xi_{l}}(\gamma)>0$, the place $\gamma$ is either empty after the $l$ th step or is filled with the symbol "•" (see Theorem $1.7 \mathrm{a})$ ). In the latter case, we have $(a, b) \in M$. Since $\mathfrak{m}$ is an ideal of $\mathfrak{n}$, we conclude that $(a, p) \in M$; this proves the statement of the lemma.

Suppose that $\gamma$ was empty after the $l$ th step; then the place $\gamma$ was also empty after the $(l-1)$ th step. By Theorem $1.7 \mathrm{a}), w_{\xi_{l-1}}(\gamma)>0$. Since the place $\xi_{l}$ was also empty after the $(l-1)$ th step, we have $w_{\xi_{l-1}}\left(\xi_{l}\right)>0$. Since $\eta=\xi_{l}+\gamma$, we see that $w_{\xi_{l-1}}(\eta)>0$. 
Since $a>b$, the place $\eta$ is filled after the $(l-1)$ th step. Theorem 1.7 implies that the place $\eta$ can be filled only with the symbol "•" (i.e., $\eta \in M$ ).

Notation and definitions 3.12. 1) By a segment $[a, b], a, b \in \mathbb{N}$, in the set of positive integers $\mathbb{N}$ we mean the set $\{i \in \mathbb{N} \mid a \leq i \leq b\}$.

2) Let $C \subset \mathbb{N}$. We say that a set $A \subset C$ is a segment in $C$ if $A$ is the intersection of a segment in $\mathbb{N}$ with $C$.

3) We introduce a relation $<$ on the set of segments: $A<B$ if and only if $i<j$ for all $i \in A$ and $j \in B$.

4) If $A=[a, b]$ and $B=[c, d]$, we introduce the relation $A \triangleleft B$ (respectively, $B \triangleright A$ ) assuming that $a=c$ and $A \subseteq B$ (respectively, $b=d$ and $B \supseteq A$ ).

Decompose $D$ and $F$ into segments:

$$
\begin{aligned}
& D=D_{1} \sqcup D_{2} \sqcup \cdots \sqcup D_{l}, \quad D_{1}<D_{2}<\cdots<D_{l}, \\
& F=F_{1} \sqcup F_{2} \sqcup \cdots \sqcup F_{l}, \quad F_{1}<F_{2}<\cdots<F_{l} .
\end{aligned}
$$

Note that

$$
h \in D_{1}<F_{1}<D_{2}<F_{2}<\cdots<D_{l}<F_{l} \triangleright\left[k, a^{(t)}\right] .
$$

For $i \in E$, let $\left\{i_{\alpha}\right\}$ be the sequence defined as follows. If $i>t$, then, as in the proof of Lemma 3.1, we put $i_{0}=i, i_{1}=w_{\xi}^{(t)}\left(i_{0}\right)$, and $i_{\alpha}=w^{(t-\alpha+1)}\left(i_{\alpha-1}\right)$, where $2 \leq \alpha \leq t$. If $i \leq t$, then $i_{\alpha}$ is defined similarly, with the replacement of $\xi$ by the smallest, with respect to $\succ$, element of $S^{[i-1]}$.

Definition 3.13. For any $i \in E$ we denote by $i^{\prime}$ the greatest number in the sequence $\left\{i_{\alpha}\right\}$ that is less than $i$. By the proof of Lemma 3.1 (see the end of a)), if $i^{\prime}=i_{\alpha}$, then $i_{\alpha}=i-\alpha+1<i$ and $i_{\alpha-1} \geq i_{\alpha-2} \geq \cdots \geq i$.

For any $i \in I_{*}$ we construct a chain

$$
i>i^{\prime}>i^{\prime \prime}>\cdots>i^{(\mu(i))}=w_{\xi *}(i) \in F_{0}
$$

in which $i^{(p)}=\left(i^{(p-1)}\right)^{\prime}$ for every $0 \leq p \leq \mu(i)$.

Lemma 3.14. 1) For different $i \in I_{*}$, the chains (3.10) do not intersect.

2) For each $b \in F_{0}$ there exists $i \in I_{*}$ such that $b=i^{(\mu(i))}$.

3) If $a \in F \cap J$, then $a=i^{(\nu)}$ for some $i \in I_{*}$ and $1 \leq \nu \leq \mu(i)$.

Proof. If $a$ is a common element of the chains (3.10) for $i_{1}$ and $i_{2}$ in $I_{*}$, then

$$
w^{(c)} \cdots w^{(a-1)}(a)=w_{\xi *}\left(i_{1}\right)=w_{\xi *}\left(i_{2}\right) .
$$

Hence, $i_{1}=i_{2}$. This proves 1 ).

Statement 2) follows from the relations $w_{\xi *}\left(I_{*}\right) \subset F_{0}$ and $\left|I_{*}\right|=h-c=\left|F_{0}\right|$ (see the proof of Lemma 3.7 and the definition of $F_{0}$ in Notation 3.91 )).

Finally, let $a \in F \cap J$ (i.e., $a \in F$ and $a \leq t$ ). Then $w_{\xi *}^{[a-1]}(a) \in F_{0}$. There exists $i \in I_{*}$ such that

$$
w_{\xi *}^{[a-1]}(a)=w_{\xi *}(i) .
$$

We have $a=w^{(a)} \cdots w_{\xi}^{(t)}(i)$, and therefore $a$ belongs to the chain (3.10) for $i$ (see the proof of Lemma 3.7).

Definitions 3.15. 1) For any $1 \leq a \leq l$ we denote

$$
\begin{aligned}
D_{[a]} & =D_{1} \sqcup \cdots \sqcup D_{a}, \\
F_{[a]} & =F_{1} \sqcup \cdots \sqcup F_{a}, \\
F_{a}^{\prime} & =\left\{i^{\prime} \mid i \in F_{a}\right\}, \\
F_{[a]}^{\prime} & =F_{1}^{\prime} \sqcup \cdots \sqcup F_{a}^{\prime} .
\end{aligned}
$$


2) Note that $k \in I_{*} \subset F_{l}$. Denote

$$
\begin{array}{ll}
F_{l 1}=\left\{i \in F_{l} \mid i<k\right\}, & F_{l 2}=\left\{i \in F_{l} \mid i \geq k\right\}, \\
F_{l 1}^{\prime}=\left\{i^{\prime} \mid i \in F_{l 1}\right\}, & F_{l 2}^{\prime}=\left\{i^{\prime} \mid i \in F_{l 2}\right\} .
\end{array}
$$

3) Denote $E_{a}=D_{a} \sqcup F_{a}$ for $1 \leq a<l$, and put $E_{l}=D_{l} \sqcup F_{l 1}$. Denote

$$
E_{[a]}=E_{1} \sqcup \cdots \sqcup E_{a} \text {. }
$$

Note that $E=E_{[l]} \sqcup F_{l 2}$ and that $E_{a}, E_{[a]}$, and $F_{0} \sqcup E_{[a]}$ are segments of positive integers, and moreover, $E_{[a]} \triangleleft E$ and $F_{0} \sqcup E_{[a]} \triangleleft[c, n]$ for any $1 \leq a \leq l$.

4) For any $1 \leq a<l$ we denote by $J_{a}$ the subset $F_{[a]}^{\prime} \sqcup D_{[b(a)]}$, where $b(a)$ is the least number such that $F_{[a]}^{\prime} \subseteq F_{[b(a)]}$ (if $b(a)=0$, we put $D_{[0]}=\varnothing$ ).

5) Let $J_{l}$ denote the subset $F_{[l-1]}^{\prime} \sqcup F_{l 1}^{\prime} \sqcup D_{[b(l)]}$, where $b(l)$ is the least number such that $F_{[p-1]}^{\prime} \sqcup F_{l 1}^{\prime} \subset F_{[b(l)]}$.

Lemma 3.16. The following is true for any $1 \leq a \leq p$ :

1) $F_{[a]}^{\prime} \subset F_{[a-1]}$;

2) $F_{[a]}^{\prime}$ is a segment in $F$;

3) $J_{a}$ is a segment of positive integers, and moreover, $J_{a} \triangleleft F_{0} \sqcup E_{[b(a)]}$;

4) if $i>E_{[a]}$ and $j \in J_{a}$, then $(i, j) \in M$;

5) the minor of the matrix $\Phi_{\mathcal{L}}$ with the system of rows $F_{a}$ and columns $F_{a}^{\prime}$, where $1 \leq a<l$, is nonzero;

6) the minor of the matrix $\Phi_{\mathcal{L}}$ with the system of rows $F_{l 1}$ (respectively, $F_{l 2}$ ) and columns $F_{l 1}^{\prime}$ (respectively, $F_{l 2}^{\prime}$ ) is nonzero.

Proof. We assume that $l>a=1$. In the general case we can argue similarly, using induction on $a$.

For $a=1$ we have $F_{[1]}=F_{1}$ and $J_{1}=F_{[1]}^{\prime}=F_{1}^{\prime}$. Let $h_{1}$ be the smallest element of $D_{2}$. Then $E_{[1]}=\left[h, h_{1}\right)$.

Denote by $g$ the greatest number such that $(i, g) \in S$ for some $i \in F_{1}$. Then $F_{1}^{\prime} \subset[c, g]$. By Lemma 3.11, $\left(h_{1}, g\right) \in M$. Since $\mathfrak{m}$ is an ideal in $\mathfrak{n}$, we have $\left[h_{1}, n\right] \times[1, g] \subset M$, which proves 4).

Now we check that

$$
F_{1}^{\prime}=[c, g] \triangleleft F_{0} .
$$

This implies statements 1), 2) and 3) for $a=1$.

a) We show that $g<h$ (recall that $h$ is the greatest element of $D_{1}$ ). Otherwise, $g \in D_{1}$ or $g>D_{1}$.

Suppose that $g \in D_{1}$. Let $(i, g)$ be the greatest, in the sense of $\succ$, element of $S^{(g)}$ such that $i \in F_{1}$. Then $w^{(g)}(i)=g \in D_{1}$, and therefore, $w^{[i-1]}(i)=w^{[g-1]} w^{(g)}(i)=$ $w^{[g-1]}(g) \geq h$, which contradicts the fact that $i \in F$.

Suppose that $g>D_{1}$. Then $w^{(g+1)} \cdots w^{(t-1)} w_{\xi}^{(t)}(t) \geq h_{1}$. Since $\left[h_{1}, n\right] \times[1, g] \subset M$, we have $w^{(c)} \cdots w^{(g)}(p)=p$ for any $p \geq h_{1}$, whence

$$
h=w_{\xi}(t)=w^{(c)} \cdots w^{(g)} w^{(g+1)} \cdots w_{\xi}^{(t)}(t) \geq h_{1} .
$$

On the other hand, $h<h_{1}$ by definition. This contradiction proves that $g<h$.

b) We conclude the proof of (3.11). We have $w_{\xi *}\left(I_{*}\right)=F_{0}=[c, h)$. On the other hand,

$$
w^{(g+1)} \cdots w_{\xi}^{(t)}\left(I_{*}\right) \subset\left(F_{0} \backslash[c, g]\right) \sqcup F_{1} \sqcup F_{\geq 2},
$$

where $F_{\geq 2}$ is the union of all $F_{p}$ with $p \geq 2$, and

$$
w^{(c)} \cdots w^{(g)}(i)=i
$$


for all $i \in F_{0}$ (see Lemma 3.63$)$ ) and all $i \in F_{\geq 2}$ ( see a)). Hence,

$$
[c, g]=w^{(c)} \cdots w^{(g)}\left(F_{1}\right)=F_{1}^{\prime}
$$

which proves (3.11).

c) We prove statements 5). Let $f_{1}=\left|F_{1}\right|$. By formula (3.11), $g=c+f_{1}-1$. We show that there exist systems $\left\{i_{(p)} \mid 0 \leq p \leq f_{1}-1\right\}$ and $\left\{j_{(p)} \mid 0 \leq p \leq f_{1}-1\right\}$ such that $\left(i_{(p)}, j_{(p)}\right) \in S$ for all $0 \leq p \leq f_{1}-1$ and

$$
F_{1}=\left\{i_{(0)}, \ldots, i_{\left(f_{1}-1\right)}\right\}, \quad F_{1}^{\prime}=[c, g]=\left\{j_{(0)}, \ldots, j_{\left(f_{1}-1\right)}\right\} .
$$

Observe that for arbitrary $i \in F_{1}$ and $j \in F_{1}^{\prime}=[c, g]$ the following is true:

I) $w^{(j)}(i)=i$ if $i \in F_{0}$ (see Lemma 3.63$)$ );

II) $w^{(j)}(i)=j \in F_{0}$ if $i \in F_{1}$ and $(i, j)$ is the greatest, in the sense of $\succ$, element of $S^{(j)}$;

III) $w^{(j)}(i) \in F_{1}$ if $i \in F_{1}$ and $i$ does not satisfy II).

Put $F_{1}=F_{10}$. For each $0 \leq p \leq f_{1}-1$ we have

$$
w^{\left(c+f_{1}-p\right)} \cdots w^{\left(c+f_{1}-1\right)}\left(F_{1}\right)=\left\{c-f_{1}-p, \ldots, c+f_{1}-1\right\} \sqcup F_{1 p},
$$

where $F_{1 p}$ is a subset of $F_{1}$ and $\left|F_{1 p}\right|=f_{1}-p$. Since

$$
F_{1}=F_{10} \supset F_{11} \supset \cdots \supset F_{1 f_{1}}=\varnothing,
$$

for any $1 \leq p \leq f_{1}-1$ there exists $i_{(p)}=F_{1 p} \backslash F_{1, p+1}$. Denote $j_{(p)}=c+f_{1}-p-1$.

Then for $j=j_{(p)}$ we have either $w^{(j)}\left(i_{(p)}\right)=j$ or $w^{(j)}\left(i_{(p)}\right) \in F_{1, p+1}$. In any case, $\left(i_{(p)}, j_{(p)}\right) \in S$, which proves 5$)$. Statement 6$)$ can be proved similarly.

Corollary 3.17. All places $(i, j)$ with $j \in J_{a}$ and $i>E_{[a]}$ are filled with zeros in the matrix $\Phi_{\mathcal{L}}$ (respectively, filled with the symbols "•" in the diagram $\left.\mathcal{D}\right)$.

Denote by $d_{i}$ and $f_{i}$ the number of elements in $D_{i}$ and $F_{i}$, respectively. Let $\nu$ be the greatest number such that $D_{[\nu]} \subset J$. Denote $d_{*}=d_{1}+\cdots+d_{\nu}$.

Proposition 3.18. Suppose $\xi$ satisfies the assumption of Case 2. Then

1) $\operatorname{deg} M_{\xi}(\lambda)=d_{*}$

2) $\operatorname{deg} M_{\xi}^{\downarrow}(\lambda) \leq d_{*}-1$, where $M_{\xi}^{\downarrow}(\lambda)$ is a minor obtained from $M_{\xi}(\lambda)$ by moving one of the rows one line down.

Proof. A) As a polynomial in $\lambda$, the minor $M_{\xi}(\lambda)$ can be written in the form

$$
M_{\xi}(\lambda)=\sum_{r=1}^{d} M_{r} \lambda^{r}, \quad d=\operatorname{deg} M_{\xi}(\lambda) .
$$

The coefficient $M_{r}$ is the sum of some minors of the matrix $\Phi_{\mathcal{L}}$ :

$$
M_{r}=\sum_{R \subset I \cap J,|R|=r} M_{R}, \quad M_{R}=M_{I \backslash R}^{J \backslash R} .
$$

A1) Show that $d \leq d_{*}$. It suffices to prove that any minor $M_{R}$ with $r=|R|>d_{*}$ equals zero. Denote $R_{i}=R \cap E_{i}$ and $r_{i}=\left|R_{i}\right|$. By assumption,

$$
r=r_{1}+\cdots+r_{\nu}>d_{1}+\cdots+d_{\nu}=d_{*} .
$$

Let $a$ be least number such that

$$
\sum_{i=1}^{a} r_{i}>\sum_{i=1}^{a} d_{i}
$$

Note that $a \leq \nu$.

We conclude the proof of A1) in each of the following cases separately: i) $1 \leq a<\nu$ or $a=\nu<l$ and ii) $a=\nu=l$. 
i) $1 \leq a<\nu$ or $a=\nu<l$. By Definitions 3.15,

$$
\begin{aligned}
\left|J_{a}\right| & =\sum_{i=1}^{a} f_{i}+\sum_{j=1}^{b(a)} d_{j}, \\
\left|E_{[a]}\right| & =\sum_{i=1}^{a} f_{i}+\sum_{j=1}^{a} d_{j} .
\end{aligned}
$$

By Lemma 3.16, we have $1 \leq b(a) \leq a-1$. Hence,

$$
\sum_{i=1}^{b(a)} r_{i} \leq \sum_{i=1}^{b(a)} d_{i}
$$

Since $J_{a} \subseteq E_{[b(a)]}$, it follows that $J_{a} \cap R \subseteq R_{1} \sqcup \cdots \sqcup R_{b(a)}$, whence

$$
\left|J_{a} \backslash R\right|=\left|J_{a}\right|-\left|J_{a} \cap R\right| \geq \sum_{i=1}^{a} f_{i}+\sum_{i=1}^{b(a)}\left(d_{i}-r_{i}\right) \geq \sum_{i=1}^{a} f_{i} .
$$

Applying inequality (3.15), we obtain

$$
\left|J_{a} \backslash R\right| \geq \sum_{i=1}^{a} f_{i}>\sum_{i=1}^{a} f_{i}+\sum_{i=1}^{a}\left(d_{i}-r_{i}\right)=\left|E_{[a]} \backslash R\right| .
$$

By Corollary 3.17, the matrix $\Phi_{\mathcal{L}}$ has zeros at all places $(i, j)$ with $j \in J_{a}$ and $i>E_{[a]}$. The minor $M_{R}$ is zero, because all its entries off the rectangle $\left(E_{[a]} \backslash R\right) \times\left(J_{a} \backslash R\right)$, where $\left|E_{[a]} \backslash R\right|>\left|J_{a} \backslash R\right|$, are zero.

ii) $a=\nu=l$. In this case $E_{[a]}$ (respectively, $J_{a}$ ) is defined in Lemma 3.143 ) (respectively, Lemma 3.145 )). Now statement A1) can be proved as in case i), with the replacement of $f_{l}$ by $f_{l 1}=\left|F_{l 1}\right|$.

A2) To conclude the proof of A), we need to show that $M_{d *} \neq 0$. By formula (3.14), any coefficient $M_{r}$ is a sum of minors $M_{R}$. The system of nonzero summands $\left\{M_{R}|| R \mid=r\right\}$ in this sum is linearly independent. Hence, $M_{r} \neq 0$ if and only if there exists a nonzero minor $M_{R}$ in the sum (3.14).

The coefficient $M_{d_{*}}$ involves the minor

$$
M_{D}=\prod_{i=1}^{l-1} M_{F_{i}}^{F_{i}^{\prime}} \cdot M_{F_{l 1}}^{F_{l 1}^{\prime}} \cdot M_{F_{l 2}}^{F_{l 2}^{\prime}}
$$

as a summand; by statements 5) and 6) of Lemma $3.16, M_{d}$ is not equal to zero. Therefore, $M_{d_{*}} \neq 0$ and $d=d_{*}$. This proves A).

B) As before, let $I$ (respectively, $J$ ) be the system of rows (respectively, columns) of the minor $M_{\xi}(\lambda)$. The system of columns for the minor $M_{\xi}^{\downarrow}(\lambda)$ is the same as for $M_{\xi}(\lambda)$, that is $J$. There exists $g \in I$ such that $g+1 \notin I$ and the system of rows $I^{\downarrow}$ for the minor $M_{\xi}^{\downarrow}(\lambda)$ coincides with $(I \backslash\{g\}) \cap\{g+1\}$.

By the definition of the system $I$, the number $g$ is the greatest in some $F_{p}$, where $\nu \leq p<l$. Then $g+1 \in D_{p+1}$. Like $M_{\xi}(\lambda)$, the minor $M_{\xi}^{\downarrow}(\lambda)$ is presented in the form

$$
M_{\xi}^{\downarrow}(\lambda)=\sum_{r=1}^{d(\downarrow)} M_{r}^{\downarrow} \lambda^{r}, \quad \text { where } d(\downarrow)=\operatorname{deg} M_{\xi}^{\downarrow}(\lambda) .
$$

Each coefficient $M_{r}^{\downarrow}$ is a sum of minors of the matrix $\Phi_{\mathcal{L}}$ :

$$
M_{r}^{\downarrow}=\sum_{R \subset I \cap J,|R|=r} M_{R}^{\downarrow},
$$


where $M_{R}^{\downarrow}$ is the minor of the matrix $\Phi_{\mathcal{L}}$ with the system of rows $I^{\downarrow} \backslash R$ and system of columns $J \backslash R$. We show that any minor $M_{R}^{\downarrow}$ equals zero if $r \geq d_{*}$. This implies the last statement of Proposition 3.18.

The case where $r>d_{*}$ can be treated much as A1). Let $r=d_{*}$. Then inequality (3.15) is true for some $1 \leq a \leq \nu$, so that this case can also be treated as A1). We need to consider the case where $d_{i}=r_{i}$ for any $1 \leq i \leq \nu$.

Let $I_{p}$ be the subsystem of rows that consists of all $i \in I$ such that $i \leq g$. By the definition of the system $I$,

$$
I_{p}=E_{1} \sqcup \cdots \sqcup E_{\nu} \sqcup F_{\nu+1} \sqcup \cdots \sqcup F_{p} .
$$

As above, we consider the subset $J_{p}$ of columns that is the union of all $F_{i}^{\prime}, 1 \leq i \leq p$, and all $D_{j}, 1 \leq j \leq b(\nu)$ (see Notation 3.154$)$ ). We obtain

$$
\left|J_{p} \backslash R\right| \geq \sum_{i=1}^{p} f_{i}+\sum_{i=1}^{\nu} d_{i}-\sum_{i=1}^{\nu} r_{i}=\sum_{i=1}^{\nu}\left(f_{i}+d_{i}\right)+\sum_{i=\nu+1}^{p} f_{i}-\sum_{i=1}^{\nu} r_{i}=\left|I_{p} \backslash R\right| .
$$

By Corollary 3.17, all places $(i, j)$ with $j \in J_{p}$ and $i>I_{p}$ are filled with zeros in the matrix $\Phi_{\mathcal{L}}$. Respectively, in the minor $M_{R}$, all places $(i, j)$ with $j \in J_{p} \backslash R$ and $i>I_{p} \backslash R$ will be filled with zeros. Hence, in the minor $M_{R}^{\downarrow}$, all places $(i, j)$ with $j \in J_{p} \backslash R$ and $i>I_{p} \backslash(R \sqcup\{g\})$ will be filled with zeros. By (3.18),

$$
\left|J_{p} \backslash R\right|>\left|I_{p} \backslash(R \sqcup\{g\})\right| .
$$

Therefore, $M_{R}^{\downarrow}=0$.

Proposition 3.19. 1) For any $\xi \in S$ the minor $M_{I, \mathcal{L}}^{J}(\lambda)$ is extremal.

2) For any $\xi \in S$ the element $P_{\xi}$ of $K\left[L C^{*}\right]$ is invariant with respect to the coadjoint representation of the group $L$.

3) Every $P_{\xi}$ can be written in the form $P_{\xi}=y_{\xi} Q_{\xi}+R_{\xi}$, where $Q_{\xi}$ and $R_{\xi}$ belong to the subalgebra in $S(\mathcal{L})$ generated by the $y_{i j}$ with $1 \leq j<t$, and the $y_{i t}$ with $(i, t) \succ \xi$.

Remark. Since $P_{\xi}$ is invariant, $Q_{\xi}$ is also invariant.

Proof. By Theorem 2.5, statement 2) follows from 1). In Case 1, the minor $M_{\xi}(\lambda)$ has zero degree. Since any root $(w(j), j)$ with $j \in J$ belongs to $S$, we have $M_{\xi}(\lambda)=P_{\xi} \neq 0$. Lemma 3.6 implies that $M_{\xi}(\lambda)$ is extremal. By Proposition 3.18 and Lemma 3.6, $M_{\xi}(\lambda)$ is extremal in Case 2.

In Case 1, statement 3) can be proved by expanding the minor $P_{\xi}$ by its last $t$ th column. To prove 3) in Case 2, it suffices to expand all minors $M_{R}$ in (3.14) for $r=d_{*}$ and $t \notin R$ by the last $t$ th column.

Theorem 3.20. The field of invariants of the coadjoint representation of the Lie algebra $\mathcal{L}$ is isomorphic to the field of rational functions of $P_{\xi}$, where $\xi \in S$.

Proof. Theorem 1.12) implies that the field of invariants of the coadjoint representation of $\mathcal{L}$ is isomorphic to the field of rational functions of $z_{1}, \ldots, z_{s}$. The proof of the theorem can be completed by induction on $1 \leq i \leq s$, with the help of Theorem 1.1 1) and Proposition 3.19 3).

Remark 3.21. In general, the polynomial $z_{i}$ that was constructed by induction in the paper [3] does not coincide with $P_{\xi_{i}}$. 
Completion of Example 1. The generators of the filed of invariants in Example 1 are of the form

$$
\begin{gathered}
P_{\xi_{1}}=y_{41}, \quad P_{\xi_{2}}=y_{62}, \quad P_{\xi_{3}}=y_{73}, \quad P_{\xi_{4}}=y_{74} y_{41}+y_{73} y_{31}, \\
P_{\xi_{5}}=\left|\begin{array}{ccc}
y_{52} & y_{53} & y_{54} \\
y_{62} & y_{63} & y_{64} \\
0 & y_{73} & y_{74}
\end{array}\right| .
\end{gathered}
$$

For all $\xi \in S$ except for $\xi_{4}$, the situation of Case 1 occurs, and $P_{\xi}$ is a minor of $\Phi_{\mathcal{L}}$. In the case of $\xi=\xi_{4}$, the minor of the characteristic matrix

$$
M_{\xi_{4}}(\lambda)=M_{2,3,4,7}^{1,2,3,4}(\lambda)=\left|\begin{array}{cccc}
y_{21} & -\lambda & 0 & 0 \\
y_{31} & y_{32} & -\lambda & 0 \\
y_{41} & y_{42} & y_{43} & -\lambda \\
0 & 0 & y_{73} & y_{74}
\end{array}\right|
$$

is extremal and $P_{\xi_{4}}$ is its leading coefficient.

\section{REFERENCES}

[1] A. A. Kirillov, Lectures on the orbit method, Nauchn. Kniga, Novosibirsk, 2002, English transl., Grad. Stud. in Math., vol. 64, Amer. Math. Soc., Providence, RI, 2004. MR2069175 (2005c:22001)

[2] _ Unitary representations of nilpotent Lie groups, Uspekhi Mat. Nauk 17 (1962), no. 4, 57-110. (Russian) MR0142001 (25:5396)

[3] A. N. Panov, On the index of some nilpotent Lie algebras, Sovrem. Mat. i Prilozhen. 60 (2008), 122-129; English transl., J. Math. Sci. 161 (2009), no. 1, 122-129. MR2676262

[4] _ The diagram method in the investigation of coadjoint orbits, Vestnik Samar. Gos. Univ. Estestvennonauchn. Ser. 2008, no. 6, 139-151. (Russian)

[5] M. V. Ignat'ev and A. N. Panov, Coadjoint orbits of the group UT( $7, K)$, Fundam. Prikl. Mat. 13 (2007), no. 5, 127-159; English transl., J. Math. Sci. (N.Y.) 156 (2009), no. 2, 292-312. MR2379743 (2008m:20075)

[6] A. N. Panov, Involution in $S_{n}$ and associated coadjoint orbits, Zap. Nauchn. Sem. S.-Peterburg. Otdel. Mat. Inst. Steklov. (POMI) 349 (2007), 150-173; English transl., J. Math. Sci. (N.Y.) 151 (2008), no. 3, 3018-3031. MR2742857

[7] J. Dixmier, Algèbres enveloppantes, Gauthier-Villars, Paris, 1974; English transl., Enveloping algebras, Grad. Stud. in Math., vol. 11, Amer. Math. Soc., Providence, RI, 1996. MR1393197 (97c:17010)

[8] B. Parshall and J.-P. Wang, Quantum linear groups, Mem. Amer. Math. Soc. 89 (1991), no. 439, 157 pp. MR.1048073 (91g:16028)

[9] F. Gavarini, Presentation by Borel subalgebras and Chevalley generations for quantum enveloping algebras, Proc. Edinb. Math. Soc. (2) 49 (2006), 291-308. MR2243788 (2007f:17026)

[10] J. G. Jantzen, Lectures on quantum groups, Grad. Stud. in Math., vol. 6, Amer. Math. Soc., Providence, RI, 1996. MR 1359532 (96m:17029)

Samara State University, Ul. Akad. Pavlova 1, Samara 443011, Russia

E-mail address: apanov@list.ru

Received 26/JAN/2009 